\title{
Myocardial Fiber Shortening in the Circumferential Direction Produces Left Ventricular Wall Thickening during Contraction
}

\author{
Takafumi Kato, ${ }^{1}$ Nobuyuki Ohte, ${ }^{1}$ Kazuaki Wakami, ${ }^{1}$ Toshihiko Goto, ${ }^{1}$ \\ Hidekatsu Fukuta, ${ }^{1}$ Hitomi Narita ${ }^{1}$ and Genjiro Kimura ${ }^{1}$ \\ ${ }^{1}$ Department of Cardio-Renal Medicine and Hypertension, Nagoya City University Graduate School of Medical \\ Sciences, Nagoya, Japan
}

When one bends the elbow by shortening of the biceps, a knot of muscle is observed in his or her upper arm, indicating that muscle shortening is converted to muscle standing in the perpendicular direction due to the incompressibility of skeletal muscle. A similar mechanism may work in the thickening process of the left ventricular (LV) wall. Although myocardial fibers of the left ventricle shorten by about $20 \%$ along the fiber direction when they contract, thickening of the LV wall during contraction often exceeds $50 \%$. Thus, the aim of the present study was to clarify the mechanism by which myocardial fiber shortening produces such remarkable thickening of the LV wall. We hypothesized that myocardial fiber shortening in the circumferential direction causes myocardial transformation perpendicular to the fiber direction, thereby producing LV wall thickening. We evaluated this hypothesis using an incompressible model of the LV wall. In 15 healthy male volunteers (38 \pm 13 years), we calculated theoretical peak thickening values of the inner and outer LV wall layers and compared them with directly measured peak thickening values using Doppler strain imaging at the corresponding areas. The theoretical peak thickening and directly measured peak thickening were $>60 \%$ in the LV inner layer. The theoretical peak thickening was correlated with the directly measured peak thickening in the inner $(r=0.75, p<0.05)$ and outer $(r=0.61, p<0.05)$ layers. We conclude that shortening of LV circumferential myocardial fiber and incompressibility of myocardium produce LV wall thickening during contraction.

Keywords: circumferential direction; Doppler strain imaging; incompressible myocardium; left ventricle; wall thickening

Tohoku J. Exp. Med., 2010, 222 (3), 175-181. (C) 2010 Tohoku University Medical Press

When one bends the elbow by shortening of the biceps, a knot of muscle is observed in his or her upper arm, indicating that muscle shortening is converted to muscle standing in the perpendicular direction due to the incompressibility of skeletal muscle (Johansson et al. 2000). Incompressibility means that myocardial volume does not change when transformation of myocardium is caused by its contraction or by the force from outside. A similar mechanism may work in the thickening process of the left ventricular (LV) wall. Although myocardial fibers of the left ventricle shorten by about $20 \%$ along the fiber direction when they contract, thickening of the LV wall during contraction often exceeds $50 \%$. This may be different from the finding observed in the skeletal muscle. The fact that $20 \%$ fiber shortening produces greater than $50 \% \mathrm{LV}$ wall thickening is puzzled. Because of the incompressibility of the myocardium (Tsuiki and Ritman 1980; Nakano et al. 1988), the cross-sectional area of a myocardial fiber increases by about $20 \%$ with its shortening. However, this finding alone cannot explain the mechanism responsible for the generation of greater than 50\% myocardial thickening. Thus, the aim of this study was to clarify the mechanism how myocardial fiber shortening is converted to such large LV wall thickness.

We have hypothesized that myocardial fiber shortening in the circumferential direction causes myocardial transformation perpendicular to the fiber direction, i.e., toward the center of LV cavity, and produces LV wall thickening. To validate this hypothesis, we calculated theoretical peak radial strain in the inner and outer layers on the $\mathrm{LV}$ wall by considering the myocardial incompressibility on the LV wall and compared them with directly measured peak radial strain values using Doppler strain imaging at the corresponding areas. The amount of myocardial thickening is called radial strain in the field of cardiac mechanics.

Received July 21, 2010; revision accepted for publication October 4, 2010. doi: 10.1620/tjem.222.175

Correspondence: Nobuyuki Ohte, M.D., Department of Cardio-Renal Medicine and Hypertension, Nagoya City University Graduate School of Medical Sciences, Mizuho-cho, Mizuho-ku, Nagoya 467-8601, Japan.

e-mail: ohte@med.nagoya-cu.ac.jp 


\section{Subjects and Methods}

Study Subjects

Study subjects were 15 healthy male volunteers (aged 24 to 55; $38 \pm 13$ years) with no history of cardiac disease or hypertension. All of the study subjects had normal sinus rhythm without intraventricular conduction disturbances on electrocardiogram. Valvular heart disease, LV wall motion abnormality, and pulmonary hypertension were not observed in any subject by two-dimensional or Doppler echocardiography. All subjects gave written informed consent to participate in the study, and the study protocol was approved by the Ethical Guidelines Committee of the Nagoya City University Graduate School of Medical Sciences.

\section{Conventional Echocardiography}

All ultrasound examinations were performed using a commercially available echocardiograph (Aplio80 ${ }^{\mathrm{TM}}$, Toshiba Medical Systems, Tokyo, Japan) with a $3-\mathrm{MHz}$ transducer. Individuals were examined at rest lying in the left lateral decubitus position. After standardized screening for cardiac disorders, M-mode echocardiographic tracings of the left ventricle were obtained at the papillary muscle level. We measured the LV end-diastolic endocardial dimension, LV septal and posterior wall thickness at end diastole, and LV epicardial diameters at end diastole and end systole (Fig. 1). From these values, we calculated the rate of epicardial circumferential length shortening $(k)$ of the left ventricle as the following equation based on the assumption that the left ventricle in the parasternal shortaxis view is circular through a cardiac cycle.

$$
k=\left(a-a^{\prime}\right) / a
$$

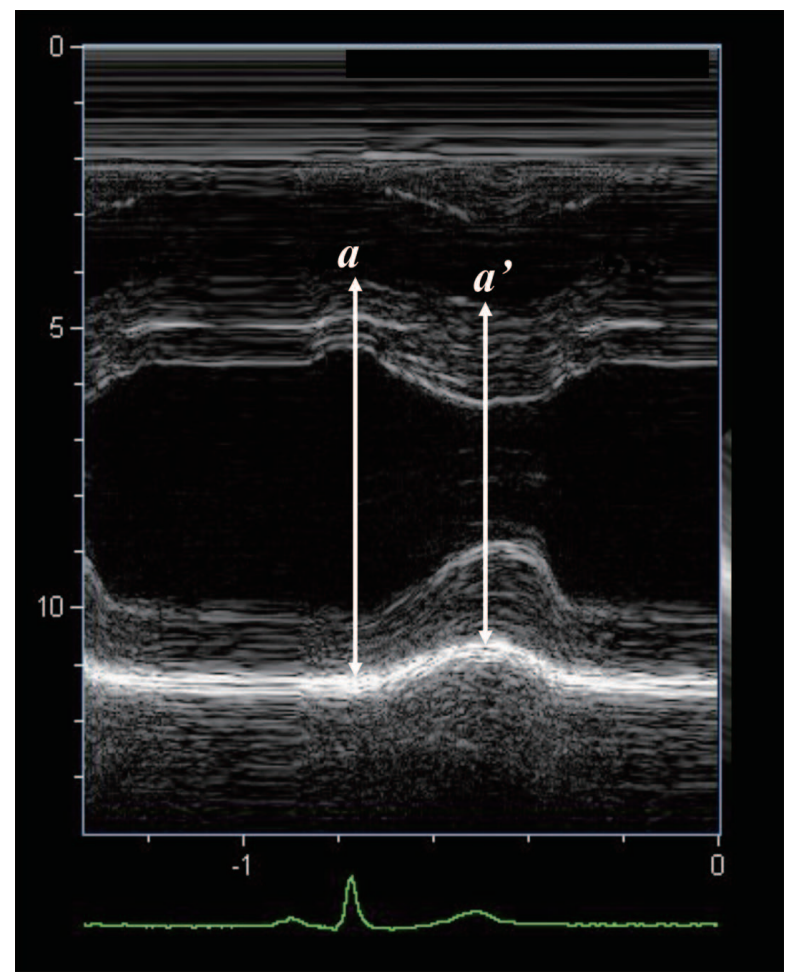

Fig. 1. M-mode tracing of the left ventricle. The left ventricular epicardial diameter appears to shorten at end systole. $a$ : left ventricular epicardial diameter at end diastole, $a$ ': left ventricular epicardial diameter at end systole.

\section{Strain Imaging}

Digital cineloops of two-dimensional tissue Doppler velocity imaging during two cardiac cycles were acquired at the papillary muscle level in the parasternal short-axis view. The frame rate was set at 74 frames/s. Thus, myocardial radial strain was analyzed every $13 \mathrm{~ms}$ in a cardiac cycle offline using an echo image analyzer (EchoAgent ${ }^{\mathrm{TM}}$, Toshiba Medical Systems). The same observer performed all analyses. For each subject, LV myocardial radial strain profiles of the subendocardium and the mid LV posterior wall were generated from myocardial velocity data. The center of contraction in the LV shortaxis image was visually and manually established at end diastole, and Doppler angle correction was used to instantaneously calculate myocardial velocities toward and away from the center of the left ventricle through an entire cardiac cycle. The center defined at end diastole was applied throughout the cardiac cycle. Myocardial velocity data sets in the cardiac cycle were then converted to myocardial displacement data sets using a tissue tracking method. Myocardial displacement data were differentiated on the basis of myocardial distance through the cardiac cycle and converted to myocardial strain data sets using the Lagrangian method (D'hooge et al. 2000).

For measurements of radial strain, we used a 3-mm length for the initial differential distance. We designated a 3-mm-square region of interest just below the endocardium to obtain the radial strain on the subendocardium. To obtain the radial strain on the mid LV wall, we designated another region of interest of the same dimensions just outside of the visually estimated centerline dividing the LV wall equidistantly. This approach to obtaining the radial strain profile using the Lagrangian method has been described previously (Maruo et al. 2007; Wakami et al. 2008). We measured peak myocardial radial strains at end systole on the strain profile, as shown in Fig. 2.

\section{Theoretical Calculation of Radial Strain}

A calculation of theoretical radial strain was performed based on the concept that myocardium is incompressible by nature (Fig. 3). Thus, the following formulas were derived under the presumption that layers shown as blue and pink in Fig. 4 remain unchanged in their areas during LV contraction.

Area of outer layer (shown in blue):

$$
\pi^{*}\left(a^{2}-b^{2}\right)=\pi *\left(a^{, 2}-b^{2}\right)
$$

Area of inner layer (shown in pink):

$$
\pi^{*}\left(b^{2}-c^{2}\right)=\pi *\left(b^{2}-c^{2}\right)
$$

From the definition, $b=(a+c) / 2$

$$
\begin{aligned}
& a^{\prime} \text { is given using } k \text { as } a^{\prime}=(1-k)^{*} a \\
& \text { From equation (1), } b^{\prime} \text { is described as } b^{\prime}=\sqrt{ }\left(a^{2}+b^{2}-a^{2}\right) \\
& \text { From equations (3) and (4), } b^{\prime} \text { is finally obtained as follows: } \\
& b^{\prime}=\sqrt{ }\left[(k-2) * k^{*} a^{2}+b^{2}\right] \\
& \text { From equation (2), } c^{\prime} \text { is described as } c^{\prime}=\sqrt{ }\left(b^{\prime 2}+c^{2}-b^{2}\right) \ldots \\
& \text { From equations (5) and (6), } c^{\prime} \text { is finally expressed as follows: } \\
& c^{\prime}=\sqrt{ }\left[(k-2) * k^{*} a^{2}+c^{2}\right]
\end{aligned}
$$

where $a$ is the radius of the outer layer, $b$ is the radius of the centerline between the inner and outer layers, $c$ is the radius of the inner layer before LV epicardial circumferential length shortening; $a$, $b$ ', and $c^{\prime}$ are these values after LV epicardial circumferential length shortening.

The peak radial strain (averaged value through the layer) gener- 


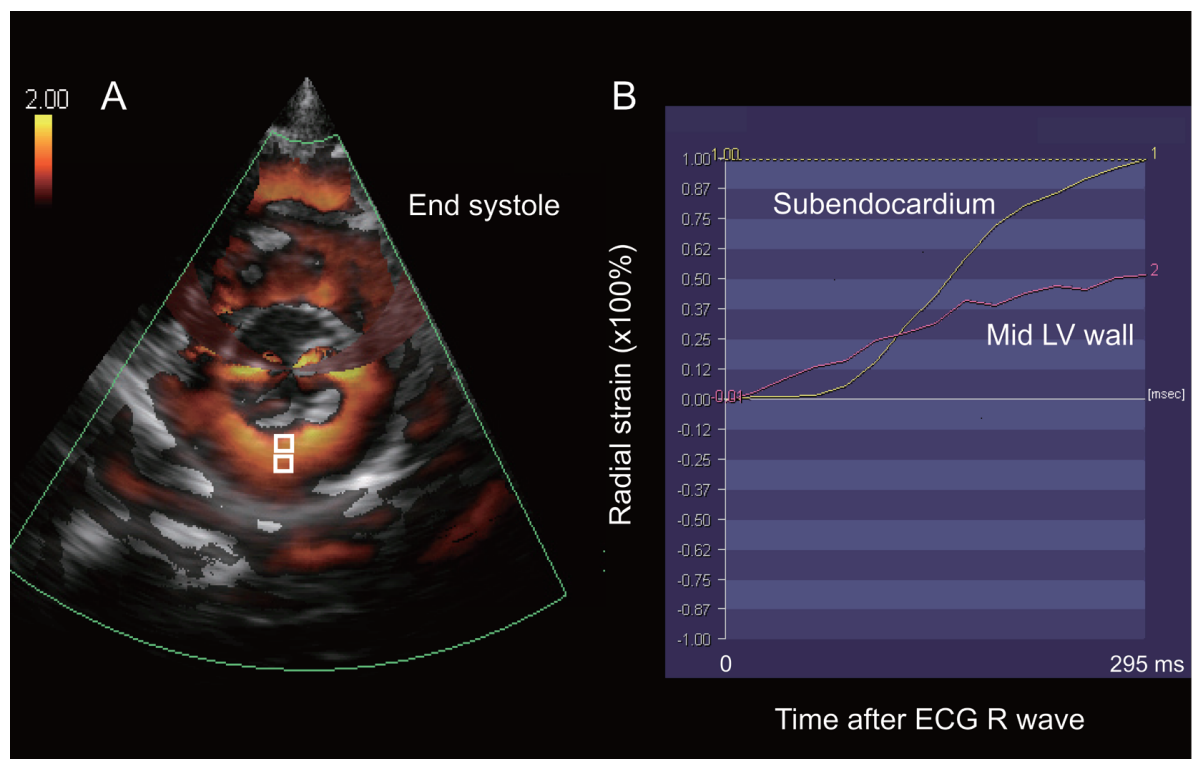

Fig. 2. Example of Doppler strain imaging of the left ventricle in the short-axis view (A). Areas with high radial strain values are shown in brighter orange as shown in the color bar on the left border. Two squares shown in white indicate the regions of interest placed at the subendocardium and just outside of the centerline between the inner and outer layers of the left ventricular wall. Representation showing the relationship between time and radial strain increase (B). In early systole, radial strain is greater on the mid left ventricular wall than on the subendocardium.

A

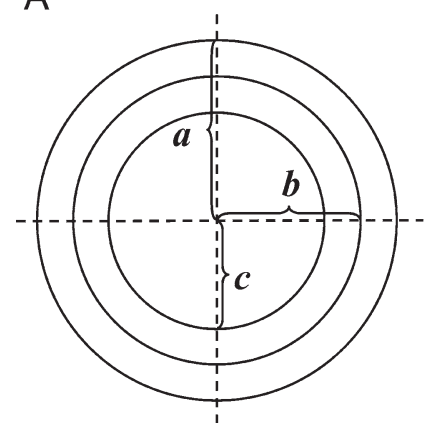

B

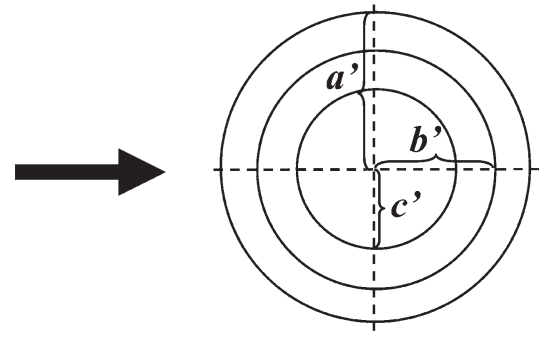

Fig. 3. Schema showing changes in radii with left ventricular epicardial circumferential length shortening. $a$ is the radius of the outer layer, $b$ is the radius of the centerline between the inner and outer layers, and $c$ is the radius of the inner layer. $a, b$, and $c$ are values obtained before epicardial circumferential length shortening (A); $a^{\prime}, b^{\prime}$, and $c^{\prime}$ are the values obtained after epicardial circumferential length shortening (B).

A

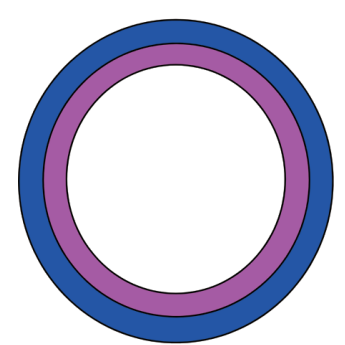

B

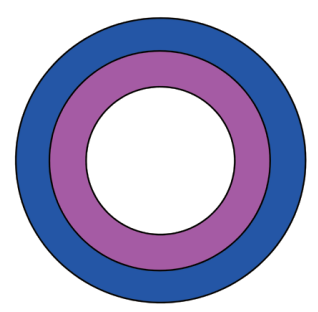

C

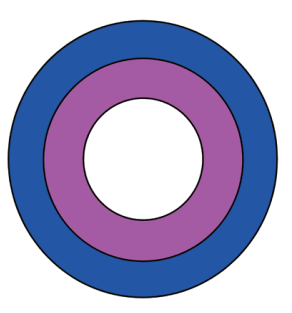

Fig. 4. Increases in left ventricular wall thickness calculated based on the incompressible nature of myocardium and epicardial circumferential length shortening. The inner layer is shown in pink, and the outer layer is shown in blue. With the increases in the rate of epicardial circumferential length shortening, left ventricular wall thickness also increased proportionally: schematic left ventricle before epicardial circumferential length shortening (A); schematic left ventricle after $10 \%$ of epicardial circumferential length shortening (B); and schematic left ventricle after $15 \%$ of epicardial circumferential length shortening $(\mathrm{C})$. 
ated by epicardial circumferential length shortening was calculated as follows:

$$
\begin{aligned}
& \text { Peak radial strain of the outer layer } \\
& \quad=\left\{\left(a^{\prime}-b^{\prime}\right)-(a-b)\right\} /(a-b) \\
& \text { Peak radial strain of the inner layer } \\
& \quad=\left\{\left(b^{\prime}-c^{\prime}\right)-(b-c)\right\} /(b-c) \ldots \ldots
\end{aligned}
$$

One can measure $a, b, c$, and $a$ ' values from M-mode tracings in each individual; $b$ ' and $c^{\prime}$ can then be calculated using the equation (7); and finally using the equations (8) and (9), one can obtain layer mean radial strains of the inner and outer layers produced by epicardial circumferential length shortening based on the incompressible nature of myocardium.

We calculated strain values using these formulas in 15 individuals. In comparing theoretical peak radial strains with actually measured peak radial strains, we uniformly added $20 \%$ to the theoretical peak radial strain values because $20 \%$ longitudinal shortening of the left ventricle causes $20 \%$ radial thickening based on the incompressible nature of myocardium. It has been noted that the LV wall in normal subjects shortens around $20 \%$ in the longitudinal direction, produced by the contraction of myocardial fibers that run longitudinally in the LV wall (Ohte et al. 2004).

\section{Statistical Analysis}

Statistical software (SPSS Version 17.0, SPSS Inc, Chicago, IL) was used for statistical analysis. Data are presented as mean \pm standard deviation. Parameters between two groups were compared using the unpaired Student's $t$ test. Relationships between two parameters were evaluated by univariate linear regression analysis.

\section{Results}

\section{Numerical Model}

Fig. 4 is a representation of our concept of LV contraction in the short axis. A higher rate of epicardial circumferential length shortening with constant initial LV epicardial and endocardial radii is predicted to produce much thicker inner and outer layers after shortening.

\section{Comparison between Theoretical and Doppler-derived Radial Strains}

The mean epicardial circumferential shortening rate $k$ was $0.168 \pm 0.02$ in our study subjects. The calculated theoretical peak radial strain was more than $60 \%$ in the inner layer; however, the value was significantly smaller than the Doppler-derived peak radial strain on the subendocardium $(60.1 \pm 10.3 \%$ vs $87.4 \pm 20.8 \%, p<0.001)$. There were no significant differences between the theoretical peak radial strain in the outer layer and the Dopplerderived peak radial strain on the mid LV wall $(41.7 \pm 7.5 \%$ vs $44.5 \pm 3.6 \%$ ) (Fig. 5). The theoretical peak radial strain on the inner layer and the Doppler-derived peak radial strain on the subendocardium correlated significantly. A significant correlation was also observed between the theoretical peak radial strain on the outer layer and the Doppler-derived peak radial strain on the mid LV wall (Fig. 6). The theoretical peak radial strain was significantly greater for the inner layer than the outer layer $(p<0.001)$.

\section{Subendocardial and Mid LV Strains in Early Systole}

If an initial increase in radial strain on the outer layer causes the subsequent increase in radial strain on the inner layer, augmentation of the actually measured radial strain may manifest on the mid LV wall rather than on the subendocardium in early systole. Indeed, in 13 of 15 study subjects, we found that the radial strain on the mid LV wall was greater than that on the subendocardium in such a phase. In all subjects, at $66 \mathrm{~ms}$ after the electrocardiographic $\mathrm{R}$ wave, the radial strain value was greater on the mid $\mathrm{LV}$ wall than on the subendocardium (11.6 \pm 6.3 vs $3.8 \pm 3.2 \%$,
A

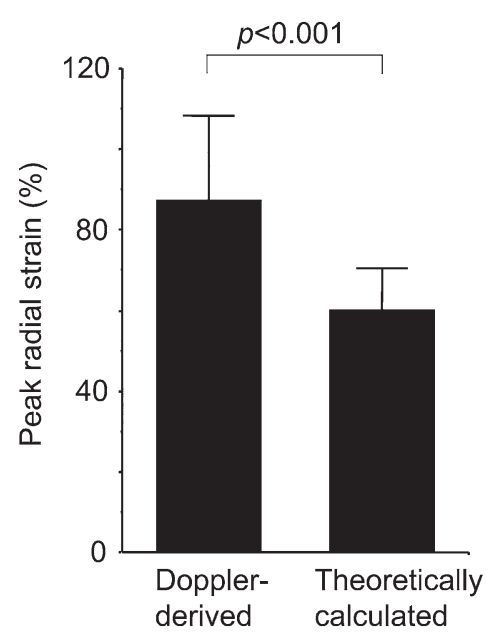

B

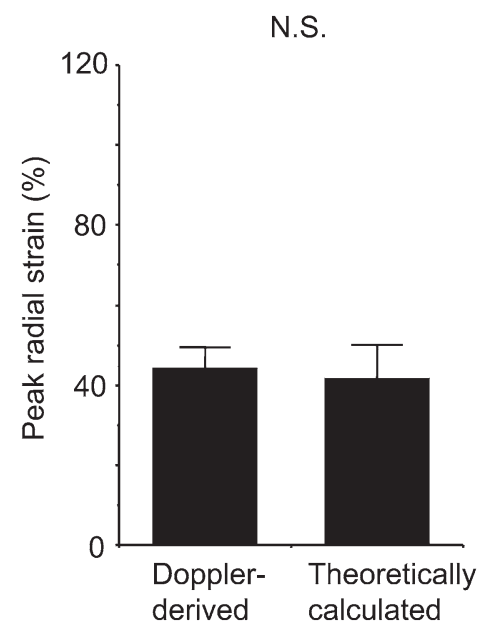

Fig. 5. Comparisons between Doppler-derived and calculated theoretical peak radial strains in the inner layer (A) and in the outer layer (B). The theoretical peak radial strain was more than $60 \%$ in the inner layer; however, the value was significantly less than the Doppler-derived peak radial strain. In contrast, there was no significant difference between the theoretical peak radial strain and the Doppler-derived peak radial strain for the outer layer. 
A

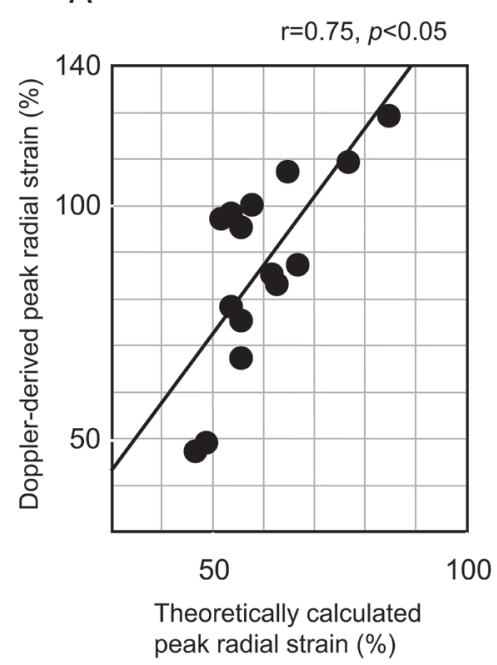

B

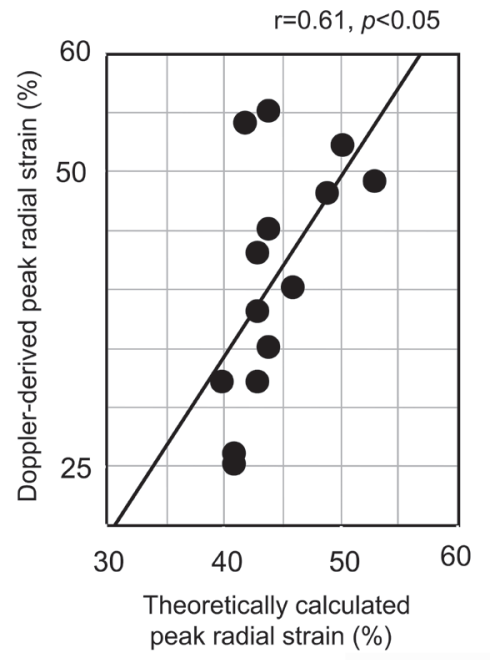

Fig. 6. Relationships between calculated theoretical peak radial strain and Doppler-derived peak radial strain in the inner (A) and outer (B) layers of the left ventricular wall. Significant correlations were observed.

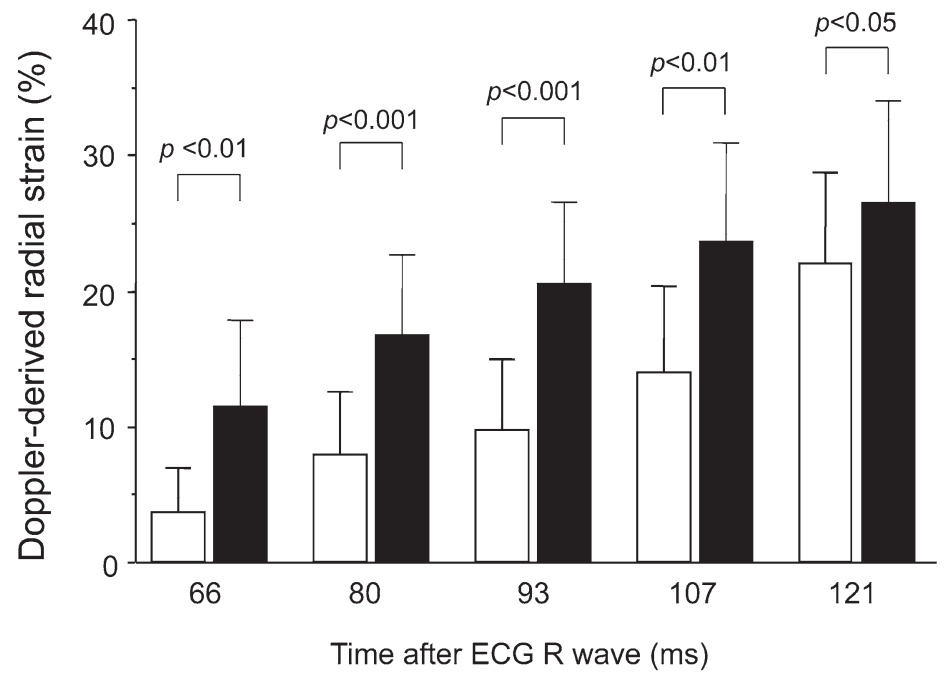

Fig. 7. Differences in Doppler-derived radial strains between subendocardium and mid left ventricular wall in early systole. Radial strains on the mid left ventricular wall were significantly greater than those on the subendocardium in early systole. White column: radial strain on the subendocardium; black column: radial strain on the mid left ventricular wall.

$p<0.01)$. Findings were similar at $80 \mathrm{~ms}(16.8 \pm 5.9$ vs 8.0 $\pm 4.6 \%, p<0.001), 93 \mathrm{~ms}(20.6 \pm 6.2$ vs $9.8 \pm 5.2 \%, p<$ $0.001), 107 \mathrm{~ms}(23.7 \pm 7.3$ vs $14.1 \pm 6.3 \%, p<0.01)$, and $120 \mathrm{~ms}(26.6 \pm 7.4$ vs $20.1 \pm 6.7 \%, p<0.05)$ (Fig. 7$)$.

\section{Discussion}

In this study, we compared the theoretical peak radial strains with actually measured peak radial strains using Doppler strain imaging. Our results demonstrate that epicardial circumferential length shortening, which is produced by myocardial fiber contraction in the circumferential direction, plays a substantial role in increasing myocardial radial strain during contraction.

The helical orientation of cardiac fibers causes integrated but complex LV contraction and induces LV wall longitudinal shortening, radial thickening, and torsional deformation (Greenbaum et al. 1981; Notomi et al. 2005; Torrent-Guasp et al. 2005). Thus, LV wall motion during contraction must be analyzed in the three orthogonal directions in clinical settings: motion along the long axis, the short axis, and circumferentially. In the context of blood ejection from the left ventricle into the aorta, LV wall motion during contraction in the direction of the short axis may be the most important of these (Costa et al. 1999), because it has the greatest strain value and contributes to decreased LV chamber volume at end systole to eject blood into the aorta.

Costa et al. (1999) found that in canine ventricular myocardium, the three-dimensional systolic mechanics alter laminar fiber architecture, referred to as a "sheet," producing significant myocardial systolic strain in the radial direction through sheet extension, sheet thickening, and sheet 
shearing, and contribute to LV wall thickening. This idea has been believed to be most reliable explanation of LV wall thickening during contraction (Senguputa et al. 2006). However, the specific mechanism by which myocardial fiber contraction is converted to sheet extension and to shear between sheets remains unidentified. Our hypothesis, based on the incompressible nature of myocardium (Tsuiki and Ritman 1980; Nakano et al. 1988) and our clinical observation that the LV epicardial circumferential length shortens during contraction, addresses how myocyte contraction along its fiber direction is converted to greater LV wall thickening in the radial direction, or radial strain. We demonstrated that peak radial strains on the inner and outer layers of the LV wall, calculated from the LV radii at end diastole and the rate of epicardial circumferential length shortening $k$, significantly correlated with peak subendocardial and mid LV wall strain values obtained using Doppler strain imaging. Maruo et al. (2007) found that LV radial strain was highest at the area just below the endocardium using Doppler strain imaging, and they observed a negative gradient in radial strain from endocardium to epicardium. In the present study, the calculated theoretical strain was higher in the inner layer than in the outer layer of the LV wall, and this finding was consistent with the results obtained with Doppler strain imaging.

The Doppler-derived peak radial strain on the subendocardium was significantly greater than the calculated theoretical peak radial strain on the inner layer. This finding may arise from the fact that Doppler-derived radial strain was obtained as an average in the 3-mm-square region of interest while the theoretical radial strain on the inner layer was computed as a layer mean value. Generally, the width of the inner layer of the LV wall was thicker than 3 $\mathrm{mm}$ even in end diastole. In late systole, the 3-mm-square region of interest did not cover the whole inner layer thickness but only the subendocardium where the highest transmural strain value is observed in the normal LV wall (Maruo et al. 2007). Both inner and outer layers become thicker in end systole than in end diastole, and the inner layer becomes much thicker than the outer layer because of the incompressible myocardium. At end systole, as noted above, the outer layer thickening was not as great as the inner layer thickening, and there was thus no significant difference between the Doppler-derived and the calculated theoretical peak radial strains.

As Figs. 2 and 7 show, in early systole, we observed greater increases of radial strain on the mid LV wall compared with those on the subendocardium. This finding is consistent with our hypothesis that epicardial circumferential length shortening caused by myocardial contraction along the circumferential direction produces the subsequent increase in radial strain in the inner layer of $\mathrm{LV}$ wall.

Recent advances in two-dimensional ultrasound speckle tracking imaging enabled us to analyze quantitatively regional myocardial motion and to calculate myocardial strain at multiple locations within the heart (Amundsen et al. 2006, 2008). However, because of the difficulty in evaluating LV radial strain on both endocardial and epicardial sides separately using this methodology, we applied the established Doppler tissue strain imaging for this purpose (Maruo et al. 2007; Wakami et al. 2008).

\section{Conclusion}

Shortening of LV epicardial circumferential length due to myocardial fiber contraction in the circumferential direction and incompressibility of myocardium produce $\mathrm{LV}$ wall thickening.

\section{References}

Amundsen, B.H., Crosby, J., Steen, P.A., Torp, H., Slørdahl, S.A. \& Støylen, A. (2008) Regional myocardial long-axis strain and strain rate measured by different tissue Doppler and speckle tracking echocardiography methods: a comparison with tagged magnetic resonance imaging. Eur. J. Echocardiogr., 10, 229237.

Amundsen, B.H., Helle-Valle, T., Edvardsen, T., Torp, H., Crosby, J., Lyseggen, E., Støylen, A., Ihlen, H., Lima, J.A.C., Smiseth, O.A. \& Slørdahl, S.A. (2006) Noninvasive myocardial strain measurement by speckle tracking echocardiography: validation against sonomicrometry and tagged magnetic resonance imaging. J. Am. Coll. Cardiol., 47, 789-793.

Costa, K.D., Takayama, Y., McCulloch, A.D. \& Covell, J.W. (1999) Laminar fiber architecture and three-dimensional systolic mechanics in canine ventricular myocardium. Am. J. Physiol. Heart Circ. Physiol., 276, H595-H607.

D'hooge, J., Heimdal, A., Jamal, F., Kukulski, T., Bijnens, B., Rademakers, F., Hatle, L., Suetens, P. \& Sutherland G.R. (2000) Regional strain and strain rate measurements by cardiac ultrasound: principles, implementation and limitations. Eur. J. Echocardiogr., 1, 154-170.

Greenbaum, R.A., Ho, S.Y., Gibson, D.G., Becker, A.E. \& Anderson, R.H. (1981) Left ventricular fibre architecture in man. Br. Heart J., 45, 248-263.

Johansson, T., Meier, P. \& Blickhan, R. (2000) A finite-element model for the mechanical analysis of skeletal muscles. $J$. Theor. Biol., 206, 131-149.

Maruo, T., Nakatani, S., Jin, Y., Uemura, K., Sugimachi, M., Ueda-Ishibashi, H., Kitakaze, M., Ohe, T., Sunagawa, K. \& Miyatake, K. (2007) Evaluation of transmural distribution of viable muscle by myocardial strain profile and dobutamine stress echocardiography. Am. J. Physiol. Heart Circ. Physiol., 292, H921-H927.

Nakano, K., Sugawara, M., Kato, T., Sasayama, S., Carabello, B.A., Asanoi, H., Umemura, J. \& Koyanagi, H. (1988) Regional work of the human left ventricle calculated by wall stress and the natural logarithm of reciprocal of wall thickness. J. Am. Coll. Cardiol., 12, 1442-1448.

Notomi, Y., Setser, R.M., Shiota, T., Martin-Miklovic, M.G., Weaver, J.A., Popović, Z.B., Yamada, H., Greenberg, N.L., White, R.D. \& Thomas, J.D. (2005) Assessment of left ventricular torsional deformation by Doppler tissue imaging: validation study with tagged magnetic resonance imaging. Circulation, 111, 1141-1147.

Ohte, N., Narita, H., Miyabe, H., Takada, N., Goto, T., Mizuno, H., Asada, K., Hayano, J. \& Kimura, G. (2004) Evaluation of whole left ventricular systolic performance and local myocardial systolic function in patients with prior myocardial infarction using global long-axis myocardial strain. Am. J. Cardiol., 94, 929-932.

Sengupta, P.P., Korinek, J., Belohlavek, M., Narula, J., Vannan, M.A., Jahangir, A. \& Khandheria, B.K. (2006) Left ventricular structure and function: basic science for cardiac imaging. $J$. 
Am. Coll. Cardiol., 48, 1988-2001.

Tsuiki, K. \& Ritman, E.L. (1980) Direct evidence that left ventricular myocardium is incompressible throughout systole and diastole. Tohoku J. Exp. Med., 132, 119-120.

Torrent-Guasp, F., Kocica, M.J., Corno, A.F., Komeda, M., Carreras-Costa, F., Flotats, A., Cosin-Aguillar, J. \& Wen, H.
(2005) Towards new understanding of the heart structure and function. Eur. J. Cardiothorac. Surg., 27, 191-201.

Wakami, K., Ohte, N., Sakata, S. \& Kimura, G. (2008) Myocardial radial strain in early diastole is useful for assessing left ventricular early diastolic function: comparison with invasive parameters. J. Am. Soc. Echocardiogr., 21, 446-451. 